\title{
Identification of Plasmodium falciparum and Plasmodium vivax on digital image of thin blood films
}

\author{
Hanung Adi Nugroho ${ }^{1}$, Made Satria Wibawa ${ }^{2}$, Noor Akhmad Setiawan ${ }^{3}$, \\ E. Elsa Herdiana Murhandarwati ${ }^{4}$, Ratna Lestari Budiani Buana ${ }^{5}$ \\ ${ }^{1,3}$ Department of Electrical Engineering and Information Technology, Faculty of Engineering, \\ Universitas Gadjah Mada, Yogyakarta, Indonesia. \\ ${ }^{2}$ Department of Information System, Faculty of Engineering, STIKOM, Bali, Indonesia. \\ ${ }^{4}$ Department of Parasitology, Faculty of Medicine, Universitas Gadjah Mada, Yogyakarta, Indonesia. \\ ${ }^{5}$ Diploma 3 of Medical Record, Vocational College, Universitas Gadjah Mada, Yogyakarta, Indonesia.
}

\section{Article Info}

Article history:

Received Sep 24, 2018

Revised Nov 25, 2018

Accepted Dec 17, 2018

\section{Keywords:}

Adaptive K-Means Clustering Plasmodium falciparum

Plasmodium vivax

Wrapper Feature Selection

\begin{abstract}
Observing presence of Plasmodium parasite of stained thick or thin blood films through microscopic examination is a gold standard for malaria diagnosis. Although the microscopic examination has been extensively used, misidentification might occur caused by human factors. In order to overcome misidentification problem, several studies have been conducted to develop a computer-aided malaria diagnosis (CADx) to assist paramedics in decisionmaking. This study proposes an approach to identify species and stage of Plasmodium falciparum and Plasmodium vivax on thin blood films collected from the Laboratory of Parasitology, Faculty of Medicine, Universitas Gadjah Mada. Adaptive k-means clustering is applied to segment Plasmodium parasites. A total of 39 features consisting of shape and texture features are extracted and then selected by using wrapper-based forward and backward directions. Classification is evaluated in two schemes. The first scheme is to classify the species of parasite into two classes. The second scheme is to classify the species and stage of parasite into six classes. Three classifiers applied are k-nearest neighbour $(\mathrm{KNN})$, support vector machine (SVM) and multi-layer perceptron (MLP). Furthermore, to facilitate the multiclass classification, one-versus-one (OVO) and one-versus-all (OVA) methods are implemented. The first scheme achieves the accuracy of $88.70 \%$ based on MLP classifier using three selected features. While the accuracy gained by the second scheme is $95.16 \%$ based on OVO and MLP classifier using 29 selected features. These results indicate that the proposed approach successfully identifies the species and stage of parasite on thin blood films and has potential to be implemented in the CADx system for assisting paramedics in diagnosing malaria.
\end{abstract}

Copyright (c) 2019 Institute of Advanced Engineering and Science. All rights reserved.

\section{Corresponding Author:}

Hanung Adi Nugroho,

Department of Electrical Engineering and Information Technology,

Faculty of Engineering, Universitas Gadjah Mada,

Jl. Grafika 2 Kampus UGM, Yogyakarta 55281, Indonesia.

Email: adinugroho@ugm.ac.id

\section{INTRODUCTION}

Malaria is one of the global diseases reported by the World Health Organisation [1]. Based on World Malaria Report 2016, there were 212 million cases found in 2015 which caused 429,000 of deaths [2]. In Indonesia, malaria is persists with higher endemicity in eastern part, such as in Papua with highest number of malaria annual parasite incidence was 31.93 malaria infection per 1,000 population in 2015 [3]. Malaria is 
caused by parasites Plasmodium parasites which are mediated by female Anopheles mosquitos. There are several species of Plasmodium namely Plasmodium falciparum ( $\mathrm{P}$. falciparum), Plasmodium vivax ( $\mathrm{P}$. vivax), Plasmodium malariae (P. malariae), Plasmodium ovale (P. ovale) and the last is Plasmodium knowlesi (P. knowlesi). In 2013, 56\% of malaria cases in Indonesia was caused by Plasmodium falciparum and $44 \%$ was caused by Plasmodium vivax [4].

Although malaria is life threatening, an early detection could save lives. Based on guidelines for the treatment of malaria, microscopic examination or rapid diagnostic test (RDT) is required to confirm malaria infection [5]. In Indonesia, microscopic examination is considered as gold standard for malaria diagnosis.

Manually, the microscopic examination consists of three steps i.e. by: 1) observing the presence of malaria parasite/Plasmodium, 2) identifying the Plasmodium species and 3) identifying the Plasmodium stage. Determination of the infecting Plasmodium species and stages are important for deciding appropriate therapy [6] as well as predicting the prognosis.

Medical laboratory technologists in either public health centres or clinics or hospitals carry out microscopic examination. Blood from finger prick is used and smeared on object glass/ slides, followed by Giemsa staining process and finally, visualisation under the microscope. In terms of diagnostic accuracy, microscopic examination has potential weaknesses that might be influenced by the laboratory personnel, ability, external distraction and experience besides smear quality, reagent or equipment used [7]. Some issues such as observation time and low-reliability result have also emerged in several laboratories in Central Java, Indonesia. Due to these problems, the examination accuracy varies with a range from 50 to $95 \%$. a situation that may lead to a wrong treatment and fatality [7].

The use of computer aided diagnosis (CADx) system can assist the paramedics in observing the presence of Plasmodium parasites to generate the quantitative analysis. It may involve the digital image processing, artificial intelligence and data mining. Moreover, the system is able to learn from knowledge of paramedics in analysing the captured image to increase the accuracy of diagnosis [8].

\section{LITERATURE REVIEW}

The early symptom of malaria is not specific and might be mimicking other virus infection e.g. headache, fatigue, body aches and fever. Misdiagnosis, late or inappropriate anti malaria therapy could lead to severity - coma, anaemia, hypoglycaemia, kidney failure, brain damage and metabolic acidosis - or even death [9]. The life cycle of malaria parasites develops in two different hosts, in humans and in female Anopheles mosquitoes as depicted in Figure 1. In the human body, the parasites undergo two cycles, exoerythrocytic and erythrocytic cycles. Merozoites as the product of exoerythrocytic cyle, invade human red blood cells, turn into trophozoite, mature and become schizont stage. Red blood cells containing schizonts are finally burst and released new merozoites that are going to invade the new red blood cells. After some cycles, some parasites turn into gametocyte stage. When a mosquito bites human having gametocytes in their blood, next transmission occurs after the gametocyte completing sporogonic cycle and generates sporozoites in the salivary fluids of the mosquito, which are ready to be injected to another human blood [10].

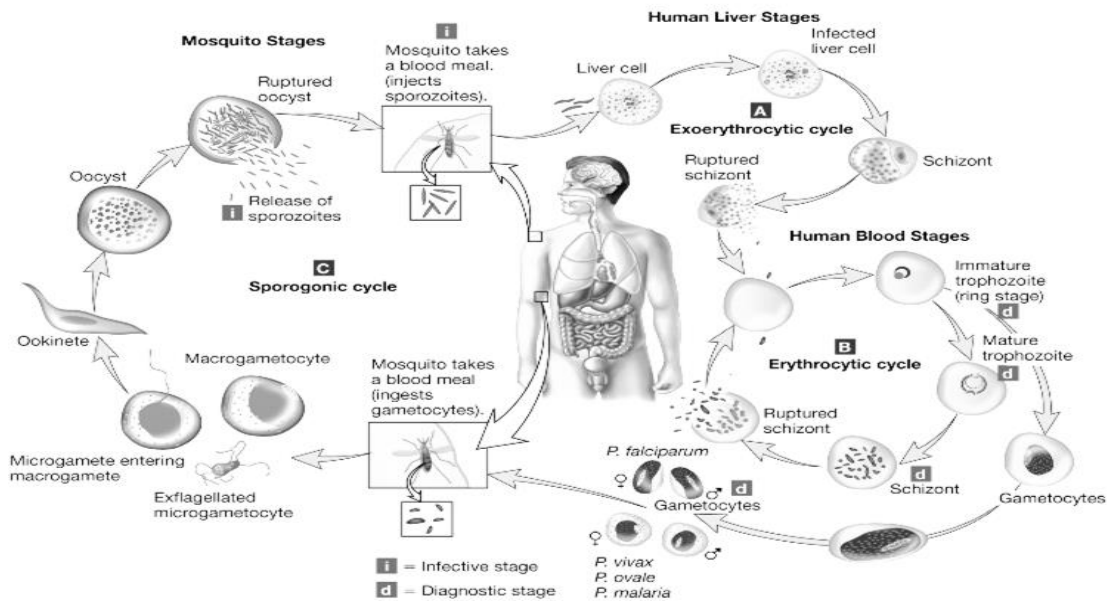

Figure 1. The life cycle of malaria parasite [10] 
Various research works have been conducted to identify malaria parasite based on CAD system. Mehrjou et al. [11] detected the red blood cells infected by malaria parasite on the thin blood films. Segmentation process was conducted on HSV colour model based on adaptive estimated threshold to separate parasite from the background. Khan et al. [12] used b-band from CIE L*a*b colour model to facilitate identification process for the presence of parasite. K-means clustering was applied in segmentation process. However, $\mathrm{k}$ value was manually determined. Moreover, this study limited only to identify the presence of parasite while the parasite species was not accomplished yet.

Savkare et al. [13] conducted a study to identify the species and stage of P. falciparum and P. vivax. Some methods were applied in pre-process such as median filter, Laplacian filter and contrast enhancement. In segmentation process, watershed was used to identify stage of parasite while Otsu thresholding used to identify its species. Two kinds of extracted features especially contour-based and histogram-based features were classified by using SVM classifier. Similar study conducted by Purnama et al. [14], but they conducted the experiment on the thick blood films. The parasite object was not obtained by segmentation process but manually cropping in the region of interest (RoI). Then, histogram-based features were extracted from RoI followed by classification process based on genetics algorithm.

Furthermore, a scheme to identify Plasmodium vivax in thin blood smear was also proposed by Akbar et al. [15]. This study was conducted with the Laboratory of Parasitology, Faculty of Medicine, Universitas Gadjah Mada. A total of 60 images used consist of 20 trophozoites, 20 gametocytes and 20 schizonts. Segmentation process was done by applying k-means clustering. However, the parameter of $\mathrm{k}$ was manually determined. A total of seven histogram-based features were used in this study.

\section{METHODOLOGY}

Based on the aforementioned literatures, there are some challenges in order to increase the performance for identifying the species and stages of malaria. The improvement of image quality is started by taking images in lossless format with higher resolution during acquisition process. Then, contrast enhancement is conducted in pre-processing. The $\mathrm{k}$ parameter of $\mathrm{k}$-means clustering used in segmentation process can be automatically determined by considering the image histogram.

Moreover, more number of features used followed by feature selection process were estimated to increase the performance of classification. The classification of species and stages of malaria parasite is a multiclass term. Most of the classifiers were developed to overcome binary class term. Therefore, OVO (oneversus-one) and OVA (one-versus-all) can be applied for optimising the classification result. The five main steps conducted in this study are described in Figure 2.

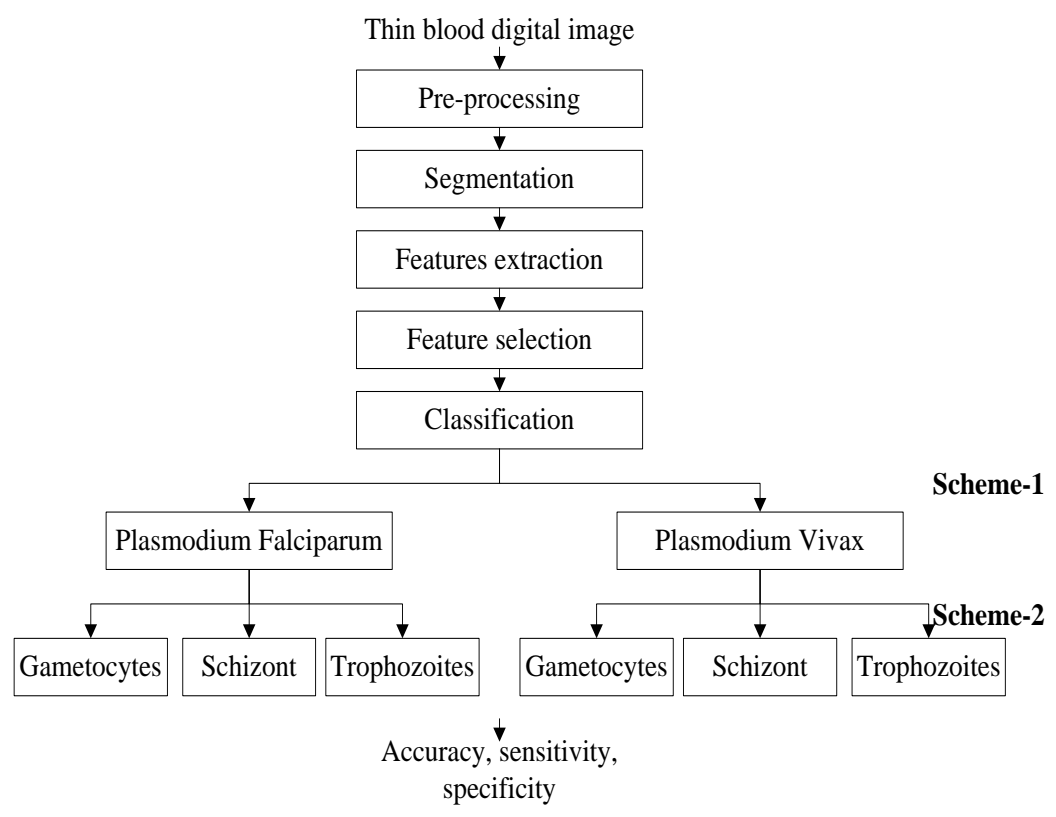

Figure 2. Block diagram of the proposed approach 


\subsection{Material and Tools}

This study uses 124 digital parasite images of thin blood films including $61 \mathrm{P}$. falciparum and $63 \mathrm{P}$. vivax. Plasmodium falciparum consisting of 24 gametocytes, 16 trophozoites and 21 schizonts. Moreover, Plasmodium vivax comprises of 23 gametocytes, 23 trophozoites and 17 schizonts as illustrated in Figure 3. The images were collected from the Laboratory of Parasitology, Faculty of Medicine, Universitas Gadjah Mada. The ground truth images were manually sketched by medical experts using a photo-editing tool. A new layer is added above original image, then medical expert marks the boundary of parasite object based on the appearance of original image. Finally, marked image is converted to binary scale.

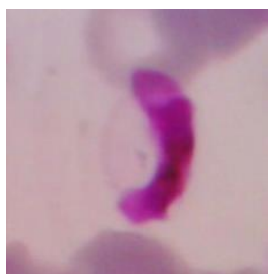

(a)

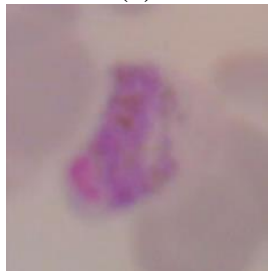

(d)

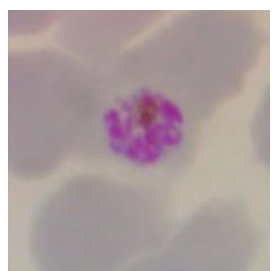

(b)

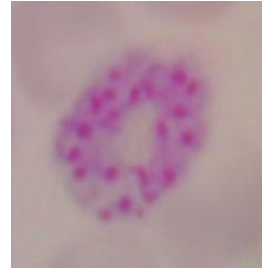

(e)

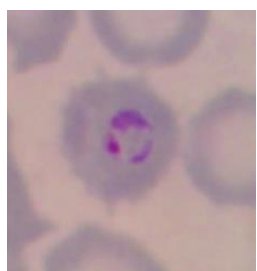

(c)

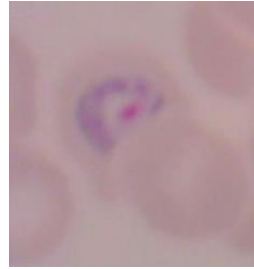

(f)

Figure 3. The images sample of P. falciparum (a) gametocytes (b) schizonts (c) trophozoites; The images of $P$. vivax (a) gametocytes (b) schizonts (c) trophozoite

\subsection{Data Acquisition}

The acquisition process involves binocular light microscope and Optilab camera. The magnification used in objective lens is 100 times which is 10 times bigger than that of the ocular lens. The resolution of original image obtained by Optilab camera is 1600x1200 pixels with lossless (bitmap) format. Each slide of blood films can generate several parasite images depending on the parasite density contained.

\subsection{Pre-processing}

Initially, the region of interest (RoI) with resolution of 250x250 pixels is obtained by cropping the area containing parasites as depicted in Figure 4. The size is considered to be covering the whole area of parasites. During this step, the haematologists validated the RoI yielded.

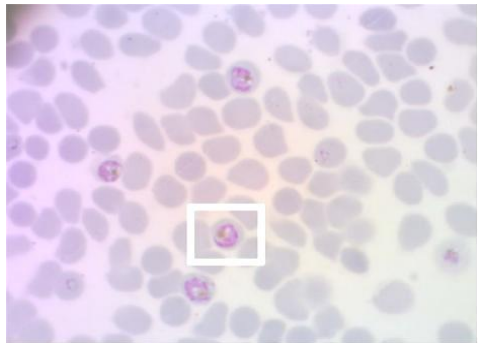

(a)

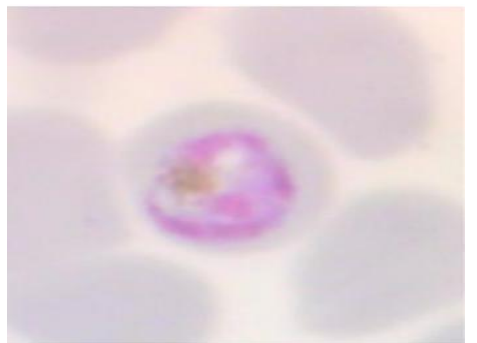

(b)

Figure 4. (a) Original image and (b) RoI image

Since the RoI image has a low contrast as presented in Figure 4 (b), hence the boundary of red blood cells and its background tends to be hard to separate. This issue is solved by applying power-law 
transformation as formulated in (1) [16]. Here, $c$ and $r$ are positive constants, while $\gamma$ (gamma) is an exponential value. The power-law transformation changes the range of image contrast to be more extensive known as gamma correction. Afterwards, the green channel is extracted to facilitate the segmentation step.

$$
s=c r^{\gamma}
$$

\subsection{Segmentation}

Segmentation is an important step before conducting feature extraction process. Segmentation aims to separate parasites from other objects, such as red blood cells, background and artefacts. The number of $\mathrm{k}$ in k-means clustering is determined by finding the significant local maxima on the green channel histogram.

Histogram is a graphical representation of the intensity values distribution [17]. The local maxima (peak of intensity) represent the centre of area, which has similar characteristic of intensity value. Opening morphological operation with structuring element size of $5 \times 5$ is then conducted for obtaining better segmentation result followed by closing operation with the structuring element size of $7 \times 7$.

\subsection{Feature Extraction}

Three kinds of features consisting of shape, texture of histogram in first order and second order are extracted. The twelve shape features are the object area, convex area, convexity, object perimeter, convex perimeter, solidity, compactness, roundness, trimness, first invariant moment, second invariant moments and third invariant moments. Texture features of histogram in first order consist of mean, standard deviation, entropy, skewness and kurtosis while that of in second order includes contrast, correlation, energy and homogeneity.

These texture features are extracted from different colour channels, particularly greyscale and green channel and saturation channel of HSV colour model. These channels are selected because the most needed information related to the characteristics of the parasite can be more clearly seen. Therefore, as of nine features are extracted from each channel, there are a total of 27 extracted texture features from three different channels.

\subsection{Feature Selection}

The wrapper needs a long time of computation to process; however, it can yield more accurate result [18]. Wrapper is chosen based on a Janecek' study [19]. Some feature selection methods are conducted to increase the classification result. In this study, wrapper is able to gain the relevant features and achieves the best classification result. Best first search (BFS) with forward and backward directions are considered during the selection process.

\subsection{Classification}

The two schemes of classification are evaluated in this study. The first scheme is to classify the species of parasite into two classes, namely P. falciparum and Plasmodium vivax. While the second scheme is to classify the stage and species of parasite at once into six classes, i.e. P. falciparum in gametocytes stage (FG), P. falciparum in schizont stage (FS), P. falciparum in trophozoite stage (FT), P. vivax in gametocytes stage (VG), P. vivax in schizont stage (VS) and P. vivax in trophozoite stage (VT). The second scheme is categorised as multiclass classification in which the number of class more than two classes. Therefore, OVO and OVA methods are used to simplify the classification process.

Various classifiers involved comprise of multi-layer perceptron (MLP), k-nearest neighbour (KNN) and support vector machine (SVM). The MLP used parameters are learning rate of 0.3 , momentum of 0.2 , the training number of 500 and one hidden layer. The adjacency parameters of KNN used for the first and the second schemes are six and two adjacencies, respectively. Meanwhile, the SVM classifier uses radial basis function (RBF) kernel. Classification is conducted in both of the full features and the selected features. Moreover, 10-folds cross validation is considered during the process.

\subsection{Evaluation and Validation}

Some parameters are used to measure the performance of the proposed approach. The segmentation process is validated by comparing the segmented parasites with that of the ground truth image. Then, the positive predictive value (PPV) as formulated in (2) is used to evaluate the performance of segmentation method [20, 21]. True positive (TP) is the number of pixels represented as parasite that are correctly segmented as parasite. While false positive (FP) is the number of non-parasite pixels segmented as parasite. 


$$
P P V=\frac{T P}{T P+F P} X 100 \%
$$

Furthermore, the performance of classification process is measured by involving some parameters as presented in Table 1. True positive (TP) is positive class correctly classified as positive, while true negative (TN) is negative class correctly classified as negative. False positive (FP) is negative class classified as positive, while false negative (FN) is negative class classified as negative. These parameters are then involved to calculate the accuracy, sensitivity and specificity as formulated from (3) to (5).

Table 1. Confusion Matrix

\begin{tabular}{ccc}
\hline Actual Class & Predicted as positive & Predicted as negative \\
\hline Positive & TP & FN \\
Negative & FP & TN \\
\hline
\end{tabular}

$$
\begin{aligned}
& \text { Accuracy }=\frac{T P+T N}{T P+F N+T N+F N} \times 100 \% \\
& \text { Sensitivity }=\frac{T P}{T P+F N} \times 100 \% \\
& \text { Specificity }=\frac{T N}{F P+T N} \times 100 \%
\end{aligned}
$$

\section{RESULTS AND ANALYSIS}

\subsection{Pre-processing}

The contrast of RoI image is enhanced based on power-law transformation to make the parasite more clearly as shown in Figure 5. Extracted green channel is then selected to facilitate the segmentation process, since the contrast between parasite and background is better than others as shown in Figure 6 .

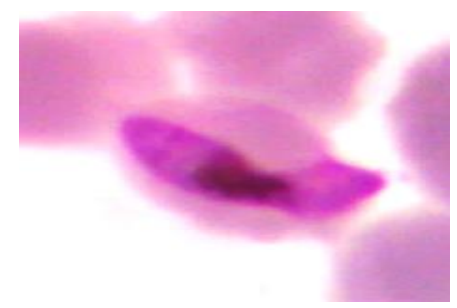

Figure 5. The images result of power-law transformation

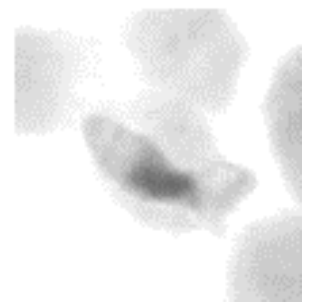

(a)

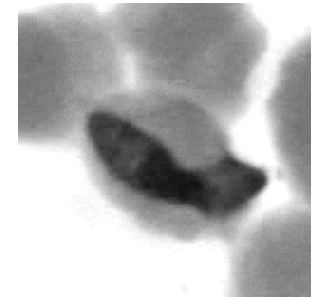

(b)

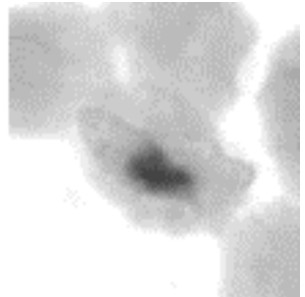

(c)

Figure 6. The extracted images of (a) red channel (b) green channel and (c) blue channel

\subsection{Segmentation}

Segmentation is conducted based on k-means clustering. The number of cluster is determined by considering the local maxima of histogram distribution. As illustrated in Figure 7, the histogram distribution of extracted green channel has the six peaks of local maxima. Thus, the intensity values that have similar characteristic are in one cluster. 


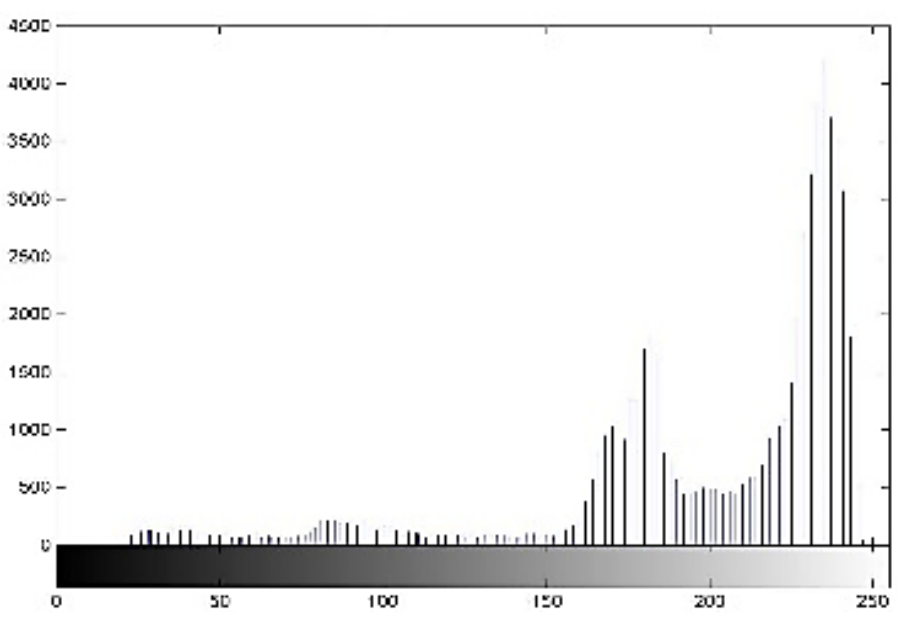

Figure 7. The sample of histogram distribution

Afterwards, the resulted image of clustering process is converted to binary scale followed by morphological operation. These results are depicted from Figure 8 (a) to Figure 8 (c). The segmented parasite is then validated to the ground truth parasite as shown in Figure 8 (d). The segmented parasites obtained by the proposed approach are covered in red line while the green line is marked as the ground truth.

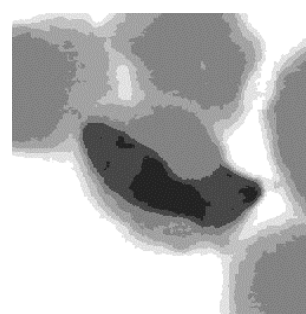

(a)

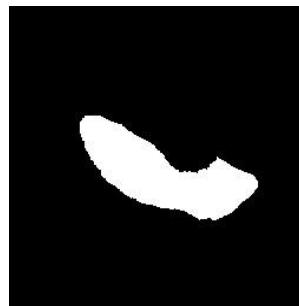

(b)

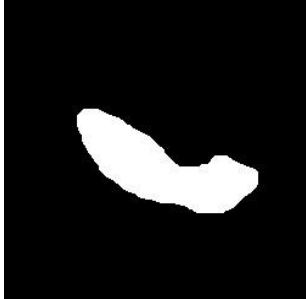

(c)

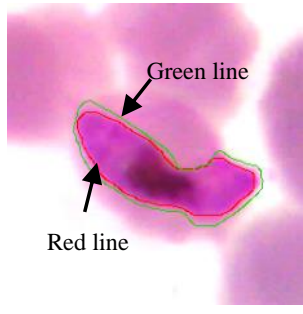

(d)

Figure 8 . The images result of (a) $k$-means clustering (b) binary scale (c) morphological (d) validated parasite

The performance of segmentation process is evaluated by calculating PPV as presented in Table 2. The higher the PPV value, the closer the segmented parasite to the ground truth. Based on Table 2, the segmentation method successfully segments parasites with the average PPV of $96.68 \%$. The smallest of PPV is obtained by P. falciparum in trophozoite stage (FT) since the parasite appears in small size and thin. Thus, the parasite is quite difficult to be segmented.

Table 2. The Evaluation Result of Segmentation Process

\begin{tabular}{ccc}
\hline Class & PPV $(\%)$ & Standard Deviation of PPV \\
\hline FG & 98.18 & 2.56 \\
FS & 97.37 & 2.83 \\
FT & 91.05 & 12.87 \\
VG & 99.22 & 1.80 \\
VS & 99.59 & 1.09 \\
VT & 94.68 & 6.21 \\
\hline
\end{tabular}

\subsection{Feature Extraction and Selection}

A total of 39 features is extracted from feature extraction process. However, not all the features may contribute to the classification process even it is potential to raise the computation time. Thus, this study uses wrapper feature selection to generate the relevant features in order to increase the classification results. 
Wrapper method is applied using forward and backward directions to find the most relevant subset features. Wrapper searches for the best subset of a feature based on the performance result of the applied classifier. Therefore, difference classifiers generate different selected features.

In the first scheme, the selected features generated by wrapper-based forward direction with SVM, KNN and MLP classifiers are 2, 2 and 3, respectively. These numbers are very small compared to the number of the 39 full features. However, the backward one is not able to reduce the number of features significantly as described in Table 3. The number of selected features for the second scheme which classifies species and stage of parasite is presented in Table 4. The wrapper based forward direction in a multiclass methods of OVO and OVA gains the significantly less number of features compared to the backward one. For the wrapper based forward direction, the smallest number of selected features is obtained by the OVO and MLP classifier with two features. Whilst the highest number of selected feature is obtained by OVO and KNN classifier with 5 features of 39 features. In wrapper based backward direction, a total of 38 features is selected by OVA and MLP classifier while the others obtain 29 selected features.

Table 3. The Number of Selected Features in the First Scheme

\begin{tabular}{ccc}
\hline Wrapper Direction & Classifier & Selected Feature \\
\hline \multirow{3}{*}{ Forward } & SVM & 2 \\
& KNN & 3 \\
& MLP & 3 \\
\multirow{3}{*}{ Backward } & SVM & 36 \\
& KNN & 36 \\
& MLP & 38 \\
\hline
\end{tabular}

Table 4. The Number of Selected Features in the Second Scheme

\begin{tabular}{cccc}
\hline Wrapper direction & Multiclass method & Classifier & Selected Feature \\
\hline \multirow{3}{*}{ Forward } & & SVM & 3 \\
& OVO & KNN & 5 \\
& & MLP & 2 \\
& & SVM & 3 \\
& & KNN & 3 \\
& & OVAP & 5 \\
& & SVM & 29 \\
Backward & OVO & KNN & 29 \\
& & MLP & 29 \\
& & SVM & 29 \\
& \multirow{3}{*}{ OVA } & KNN & 29 \\
& & MLP & 38 \\
\hline
\end{tabular}

\subsection{Classification}

In this study, two schemes of classification are evaluated. The first scheme classifies the parasite into two classes based on its species while the second scheme classifies the parasite into six classed based on its species and stage. During the process, the 10-folds cross is involved. The data is divided into ten sections with the equal composition; one section as training data and the other sections as testing data. This process is repeated 10 times until all sections have undergone as training and testing data.

\subsubsection{Scheme-1: Parasite Species Classification}

The process of species classification is carried out in the full features and also in the selected features. The OVO and OVA methods are not conducted because the species classification is binary classification with two classes consisting of $\mathrm{P}$. falciparum and $\mathrm{P}$. vivax. Table 5 presents the classification results of the first scheme on the 39 full features including of accuracy, sensitivity and specificity. The sensitivity and specificity are calculated separately between P. falciparum and P. vivax to find out the performance of classification in recognising true positive and true negative of each species.

As shown in Table 5, the lowest accuracy is obtained by the SVM classifier. It means that the SVM classifier is not proper in recognising the parasite species. KNN classifier generates better results than SVM classifier, but the accuracy rate is still lower than that of MLP classifier. KNN classifier has better performance in recognising $\mathrm{P}$. falciparum with the sensitivity of $90.16 \%$ than that of $\mathrm{P}$. vivax with the sensitivity of $49.21 \%$. Most of the species misclassifications are coming from P. vivax which is recognised as P. falciparum. Generally, the best classification results using the 39 full features are obtained by MLP 
classifier with the accuracy, sensitivity of P. falciparum and sensitivity of P. vivax at $80.00 \%, 83.61 \%$ and $76.19 \%$, respectively.

Table 5. Two Class Classification without Feature Selection

\begin{tabular}{cccccc}
\hline Classifier & $\begin{array}{c}\text { Accuracy } \\
(\%)\end{array}$ & $\begin{array}{c}\text { Sensitivity of } P . \\
\text { falciparum } \\
(\%)\end{array}$ & $\begin{array}{c}\text { Specificity of } P . \\
\text { falciparum }(\%)\end{array}$ & $\begin{array}{c}\text { Sensitivity of } P . \\
\text { vivax }(\%)\end{array}$ & $\begin{array}{c}\text { Specificity of } P . \\
\text { vivax }(\%)\end{array}$ \\
\hline SVM & 47.63 & 39.34 & 48.61 & 55.56 & 39.34 \\
KNN & 69.69 & 90.16 & 83.78 & 49.21 & 90.16 \\
MLP & 80.00 & 83.61 & 82.76 & 76.19 & 83.61 \\
\hline
\end{tabular}

Table 6 presents the performance of classification process by using selected features. In both forward and backward directions, the SVM classifier on the selected features obtains better accuracy than that of the 39 full features. However, the sensitivity rate of P. falciparum and P. vivax is not promising yet. For the KNN classifier, better accuracy is obtained by forward direction at $81.45 \%$. However, most of P. vivax is classified as $\mathrm{P}$. falciparum indicated by the low sensitivity rate of $\mathrm{P}$. vivax at $65.08 \%$. The best performance of classification is achieved by MLP classifier using forward direction with the accuracy, sensitivity of $\mathrm{P}$. falciparum and sensitivity of P. vivax at $88.70 \%, 93.44 \%$ and $84.13 \%$, respectively. There is a significant increase of classification results compared to that of the 39 full features. These results also indicate that the MLP classifier is able to recognise the P. falciparum and P. vivax.

Table 6. Two Class Classification with Feature Selection

\begin{tabular}{ccccccc}
\hline $\begin{array}{c}\text { Wrapper } \\
\text { direction }\end{array}$ & Classifier & $\begin{array}{c}\text { Accuracy } \\
(\%)\end{array}$ & $\begin{array}{c}\text { Sens. of } P \text {. falciparum } \\
(\%)\end{array}$ & $\begin{array}{c}\text { Spec. of } P \text {. } \\
\text { falciparum }(\%)\end{array}$ & $\begin{array}{c}\text { Sens. of } P . \\
\text { vivax }(\%)\end{array}$ & $\begin{array}{c}\text { Spec. of } P . \\
\text { vivax }(\%)\end{array}$ \\
\hline \multirow{3}{*}{ Forward } & SVM & 78.22 & 91.80 & 89.13 & 65.08 & 91.80 \\
& KNN & 81.45 & 98.36 & 97.62 & 65.08 & 98.36 \\
& MLP & 88.70 & 93.44 & 92.98 & 84.13 & 93.44 \\
& & & & & & \\
Backward & SVM & 76.61 & 65.57 & 72.37 & 87.30 & 65.57 \\
& KNN & 72.58 & 91.80 & 87.18 & 53.97 & 91.80 \\
& MLP & 87.09 & 91.80 & 91.23 & 82.54 & 91.80 \\
\hline
\end{tabular}

*Sens: sensitivity; Spec: specificity

\subsubsection{Scheme-2: Parasite Species and Stage Classification}

The second scheme is conducted to classify the species and stage of parasite at once. The performance of classification on the 39 full features is displayed in Table 7. The average values of accuracy, sensitivity and specificity resulted by SVM classifier are $73.39 \%, 17.48 \%$ and $83.51 \%$, respectively. The lowest average of sensitivity states that SVM classifier is not able to recognise the true positive class. Basically, the SVM classifier is merely appropriate for binary classification.

\begin{tabular}{cccc}
\multicolumn{4}{c}{ Table 7. Six Class Classification without Feature Selection } \\
\hline Classifier & $\begin{array}{c}\text { Average of } \\
\text { Accuracy (\%) }\end{array}$ & $\begin{array}{c}\text { Average of } \\
\text { Sensitivity (\%) }\end{array}$ & $\begin{array}{c}\text { Average of } \\
\text { Specificity (\%) }\end{array}$ \\
\hline SVM & 73.39 & 17.48 & 83.51 \\
KNN & 87.09 & 60.68 & 92.22 \\
MLP & 94.62 & 82.92 & 96.78 \\
\hline
\end{tabular}

For the KNN classifier, the average of accuracy and the average of specificity are respectively at $87.09 \%$ and $92.22 \%$. However, the average of sensitivity is not satisfied since the rate merely at $60.68 \%$. The MLP classifier achieved the best performance of classification with the average of accuracy, sensitivity and specificity of $94.62 \%, 82.92 \%$ and $96.78 \%$.

The classification results using selected features are presented in Table 8 . The multiclass methods especially OVO and OVA are considered during the process to improve the classification performance. The best performance of classification is yielded by using the selected features of wrapper-based backward direction followed by OVO and MLP classifier. The average of accuracy, sensitivity and specificity achieved are $95.16 \%, 84.02 \%$ and $97.09 \%$, respectively. Whilst the performance results obtained by others are not significantly different. 
Table 8. Six Class Classification with Feature Selection and Multiclass Scheme

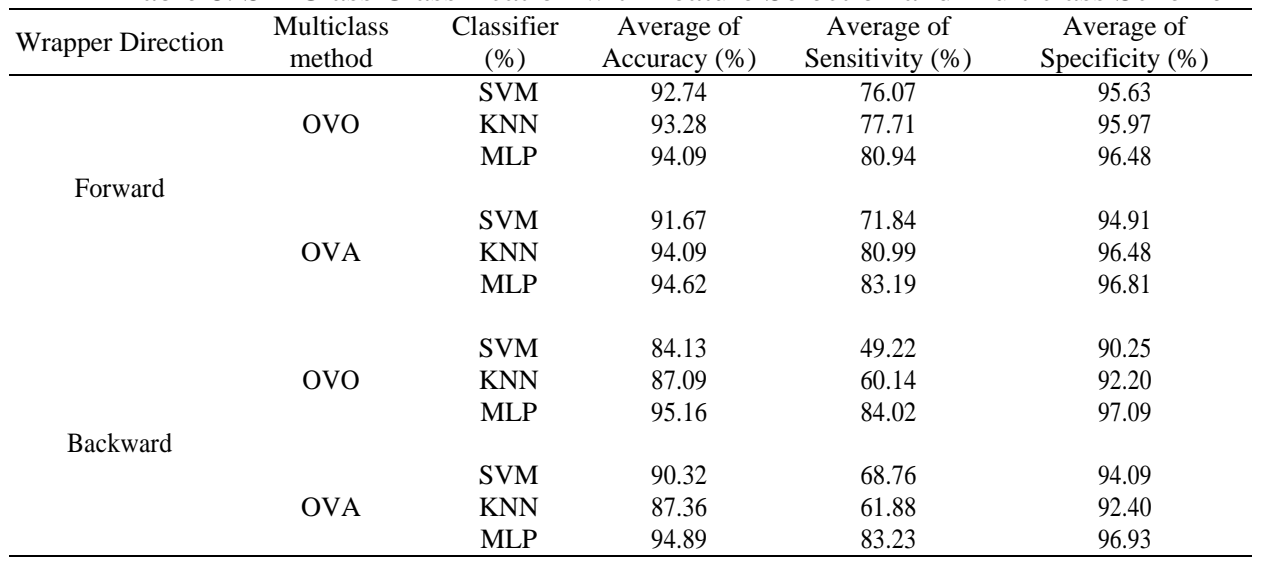

\section{CONCLUSION}

An identification approach of Plasmodium parasite on digital image of thin blood films has been proposed. Firstly, the parasite is segmented by using adaptive k-means clustering on the extracted green channel which has enhanced by applying the power-law transformation. The segmentation method is able to segment parasite properly assured by average PPV of $96.86 \%$.

Afterwards, two schemes of classification are evaluated on several relevant features selected by wrapper method. The first scheme classifies the parasite species into two classes, namely P. falciparum and P. vivax. The best classification performance of the first scheme is achieved by using three selected features of wrapper-based forward direction followed by MLP classifier with the accuracy, sensitivity of P. falciparum and sensitivity of P. vivax at $88.70 \%, 93.44 \%$ and $84.13 \%$, respectively.

Furthermore, the second scheme classifies the species and stage of parasite into six classes. The two methods of multiclass classification especially OVO and OVA are involved during the process. In this scheme, the best performance of classification is gained by using 29 selected features of wrapper-based backward direction followed by OVO and MLP classifier. The average of accuracy, sensitivity and specificity achieved are $95.16 \%, 84.02 \%$ and $97.09 \%$, respectively.

Generally, the identification of parasite is successfully conducted in both the first and the second schemes. These achievements indicate that the proposed approach has a potential to be implemented as part of the development a computerised aided malaria diagnosis system for assisting the paramedics. For further study, the expansion of the proposed approach will be conducted to recognise the other objects such as the white blood cells, artefacts or other parasites in the blood cells. Moreover, the authors will consider more features to identify more species and stages of the Plasmodium parasites.

\section{ACKNOWLEDGEMENTS}

This study is funded by Directorate General of Higher Education, Ministry of Research, Technology and Higher Education, Republic of Indonesia. The authors would also like to acknowledge the Intelligent Systems research group members in the Department of Electrical Engineering and Information Technology, UGM, for great sharing and discussion.

\section{REFERENCES}

[1] W. H. Organization, "Handbook: Integrated Management of Childhood Illness [IMCI]," Geneva: WHO, pp. 23950,2005 .

[2] W. H. Organisation, "World Malaria Report 2016," Geneva, Switzerland, 2016.

[3] P. K. K. R. Indonesia, "InfoDATIN Malaria," Pusdatin, Indonesia 2442-7659, 2016.

[4] W. H. Organisation, "World Malaria Report 2015," Geneva, Switzerland, 2015.

[5] W. H. Organisation, "Guidelines for the treatment of malaria," 3rd ed. Genewa, Switzerland, 2015.

[6] F. B. Tek, A. G. Dempster, and I. Kale, "Parasite detection and identification for automated thin blood film malaria diagnosis," Computer vision and image understanding, vol. 114, pp. 21-32, 2010.

[7] S. Tuti and W. Worowijat, "Situasi Mikroskopis Malaria di Berbagai Laboratorium Daerah Endemik, Tahun 2006," Buletin Penelitian Sistem Kesehatan, vol. 12, 2009.

[8] G. Dougherty, Medical image processing: techniques and applications: Springer Science \& Business Media, 2011.

[9] W. H. Organisation, "World Malaria Report 2014," 3rd edition ed. Genewa, Switzerland, , 2014. 
[10] J. A. Jackman and J. K. Olson, "Mosquitoes and the Diseases they Transmit," Texas FARMER Collection, 2002.

[11] A. Mehrjou, T. Abbasian, and M. Izadi, "Automatic malaria diagnosis system," in 2013 First RSI/ISM International Conference on Robotics and Mechatronics (ICRoM), 2013, pp. 205-211.

[12] N. A. Khan, H. Pervaz, A. K. Latif, and A. Musharraf, "Unsupervised identification of malaria parasites using computer vision," in 2014 11th International Joint Conference on Computer Science and Software Engineering (JCSSE), 2014, pp. 263-267.

[13] S. Savkare and S. Narote, "Automated system for malaria parasite identification," in 2015 International Conference on Communication, Information \& Computing Technology (ICCICT), 2015, pp. 1-4.

[14] I. K. E. Purnama, F. Z. Rahmanti, and M. H. Purnomo, "Malaria parasite identification on thick blood film using genetic programming," in 2013 3rd International Conference on Instrumentation, Communications, Information Technology, and Biomedical Engineering (ICICI-BME), 2013, pp. 194-198.

[15] H. A. Nugroho, S. A. Akbar, and E. E. H. Murhandarwati, "Feature extraction and classification for detection malaria parasites in thin blood smear," in 2015 2nd International Conference on Information Technology, Computer, and Electrical Engineering (ICITACEE), 2015, pp. 197-201.

[16] C.-M. Tsai, "Adaptive local power-law transformation for color image enhancement," Applied Mathematics \& Information Sciences, vol. 7, p. 2019, 2013.

[17] M. Tian, Q. Yang, A. K. Maier, I. Schasiepen, N. Maass, and M. Elter, "Automatic Histogram-Based Initialization of K-Means Clustering in CT," in Bildverarbeitung für die Medizin, 2013, pp. 277-282.

[18] M. Gutlein, E. Frank, M. Hall, and A. Karwath, "Large-scale attribute selection using wrappers," in CIDM'09. IEEE Symposium on Computational Intelligence and Data Mining, 2009, 2009, pp. 332-339.

[19] A. Janecek, W. Gansterer, M. Demel, and G. Ecker, "On the relationship between feature selection and classification accuracy," in New Challenges for Feature Selection in Data Mining and Knowledge Discovery, 2008, pp. $90-105$.

[20] J. Gatc, F. Maspiyanti, D. Sarwinda, and A. M. Arymurthy, "Plasmodium parasite detection on red blood cell image for the diagnosis of malaria using double thresholding," in 2013 International Conference on Advanced Computer Science and Information Systems (ICACSIS), 2013, pp. 381-385.

[21] A. A. Nasir, M. Mashor, and Z. Mohamed, "Segmentation based approach for detection of malaria parasites using moving k-means clustering," in 2012 IEEE EMBS Conference on Biomedical Engineering and Sciences (IECBES), 2012, pp. 653-658.

\section{BIOGRAPHIES OF AUTHORS}

H.A. Nugroho completed his Bachelor degree in Electrical Engineering (2001) from the
Universitas Gadjah Mada, Yogyakarta, Indonesia and Master degree in Biomedical
Engineering (2005) from The Univ. of Queensland, Brisbane, Australia. In 2012, he
received his PhD in Electrical and Electronic Engineering from the Universiti Teknologi
Petronas (UTP), Malaysia. Currently, he is an Assistant Professor and also a Vice Head of
Department of Electrical Engineering and Information Technology, Faculty of Engineering,
Universitas Gadjah Mada (UGM). His current research interests include biomedical signal
and image processing and analysis, computer vision, medical instrumentation and pattern
recognition.




\begin{tabular}{|l|l|} 
& $\begin{array}{l}\text { E.E.H. Murhandarwati completed her Bachelor degree (1993) and Master degree (1996) in } \\
\text { Faculty of Medicine, Universitas Gadjah Mada. In 2011, she received her PhD in Medical } \\
\text { Science Monash University. Currently, she is a Lecturer in the Department of Parasitology, } \\
\text { Faculty of Medicine, Universitas Gadjah Mada (UGM). Her research interest is tropical } \\
\text { medicine. }\end{array}$ \\
\hline $\begin{array}{l}\text { R.L. Budiani completed her Bachelor degree in Computer Science (2009) from } \\
\text { the Universitas Gadjah Mada, Yogyakarta, Indonesia and Master degree in } \\
\text { Biomedical Engineering (2015), from the University of Queensland, Brisbane, } \\
\text { Australia. She is a Research Assistant at the Department of Electrical } \\
\text { Engineering and Information Technology, Faculty of Engineering, Universitas } \\
\text { Gadjah Mada, Indonesia. }\end{array}$ \\
\hline
\end{tabular}

